\title{
SÍNDROME DE KASABACH - MERRIT: RELATO DE CASO
}

\author{
ARAÚJO, M. E. A. M. ${ }^{1}$ \\ TOLEDO, D. O. ${ }^{1}$ \\ SPIRLANDELI, A. M. ${ }^{2}$ \\ PERENCINI, A. B. ${ }^{2}$ \\ BRENTINI, B. C. ${ }^{1}$ \\ CORADIN, B. B. ${ }^{1}$ \\ PIMENTA, A. E. C. ${ }^{1}$
}

\begin{abstract}
RESUMO:A síndrome de Kasabach - Merrit (SKM) caracteriza-se pela presença de hemangioma associado à trombocitopenia, anemia hemolítica e coagulopatia consumptiva. Apesar de rara, deve ter seu diagnóstico e terapia instituídos rapidamente devido à sua elevada gravidade, podendo evoluir para ocorrência de sangramentos como petéquias, equimoses, hematomas espontâneos e até mesmo ao óbito. O presente trabalho tem como objetivo relatar o caso de um lactente que apresentou um hemangioma em região axilar e escapular de membro superior esquerdo, de rápida evolução, bem como a sua resposta ao tratamento instituído, após análise de prontuário e realizar revisão acerca da literatura disponível sobre a patologia descrita.
\end{abstract}

Palavras-chave: Hemangioma, Tratamento, Corticoides

SUMMARY: Kasabach - Merrit syndrome is characterized by the presence of hemangioma associated with thrombocytopenia, hemolytic anemia and consumptive coagulopathy. Although rare, it should have diagnosis and therapy instituted quickly due to due to the high severity of the case, and may progress to hemorrhage with petechiae, bruising, spontaneous bruising and even hearing. The aim of this study is to report the case of an infant showing a rapidly evolving axillary and scapular hemangioma of a left upper limb, as well as its response to the treatment instituted, following a chart review of the available literature on recorded pathology.

Keywords: Hemangioma, Treatment, Corticoids

\section{INTRODUÇÃO}

Hemangiomas são alterações vasculares que podem ser de origem benigna ou maligna, com curso patológico evidenciando formação tumoral com proliferação de células endoteliais e evolução normalmente autolimitado. O desenvolvimento mais comum do hemangioma é durante a infância (primeiros dias e meses de vida) não necessitando de tratamento específico. No entanto, em algumas situações, como seguimento inadequado ou evolução rápida para grave, o quadro pode se complicar e desencadear outros que necessitam de mais cuidado (SOCIEDADE BRASILEIRA DE PEDIATRIA , 2018).

Uma das complicações mais raras e com desfechos mais severos é a síndrome de Kasabach-Merrit, fenômeno esse que teve seu primeiro relato em 1940 e sendo ainda uma patologia que necessita de estudos mais consistentes para confirmar sua etiologia e fisiopatogenia. Contudo, pesquisadores a definem pela presença de um hemangioma de aumento

\footnotetext{
${ }^{1}$ Santa Casa de Misericórdia de Franca.

${ }^{2}$ Universidade de Franca (UNIFRAN)
} 
rápido, associado à plaquetopenia grave, anemia hemolítica microangiopática e coagulopatia de consumo (HAISLEY-ROYSTER et al., 2002).

É uma síndrome rara e corresponde em média a $1 \%$ dos tumores vasculares ocorridos na infância (RYAN et al., 2009) e tem como predomínio mais frequente a faixa pediátrica, principalmente durante as primeiras 5 semanas de vida (YADAV et al., 2011), com prevalência maior no sexo feminino, entre caucasianos e prematuros (BRONDANI et al., 2018). Pode estar associada ao hemangioendotelioma Kaposiforme (90\%), como também ao hemangioma congênito e infantil, além do seu desenvolvimento de forma isolada (MUÑOZ et al., 2018).

O tratamento para SKM ainda não está estabelecido, sendo ultimamente empregado o uso de corticoide sistêmico, como prednisolona, além do advento de terapias mais novas, como propranolol (BONINI; BELLODI; SOUZA, 2011).

Com isso, este trabalho tem como objetivo relatar o caso de um lactente com apresentação de hemangioma em região axilar e escapular, evoluindo de maneira rápida, assim como sua resposta ao tratamento instituído, além de realizar uma breve revisão da literatura disponível acerca da patologia descrita.

\section{RELATO DE CASO}

Este trabalho foi submetido ao Comitê de Ética em Pesquisa sob o número CAAE: 13140319.8.0000.5438 e aprovado em 20 de maio de 2019.

Paciente K.S.M. admitido na Santa Casa de Franca em 01 de outubro de 2018, devido aparecimento de edema em membro superior direito no dia 25 de setembro de 2018. Cuidadora relatava aparecimento súbito de edema violáceo em ombro esquerdo que se espalhou por todo o MSE, quando procurou o serviço médico e foi indicado Raio X, que não mostrou alteração e, encaminhado para internação e investigação diagnóstica para avaliação.

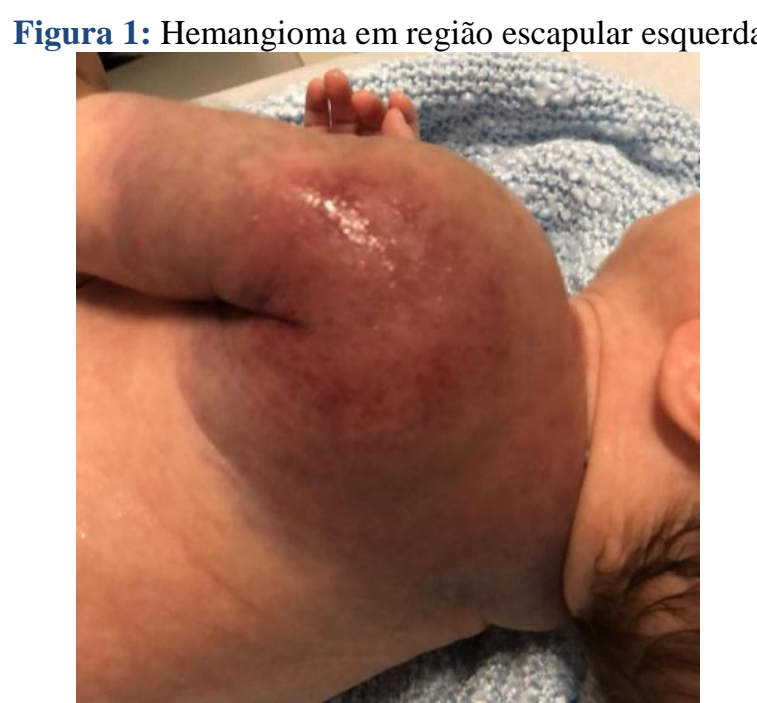




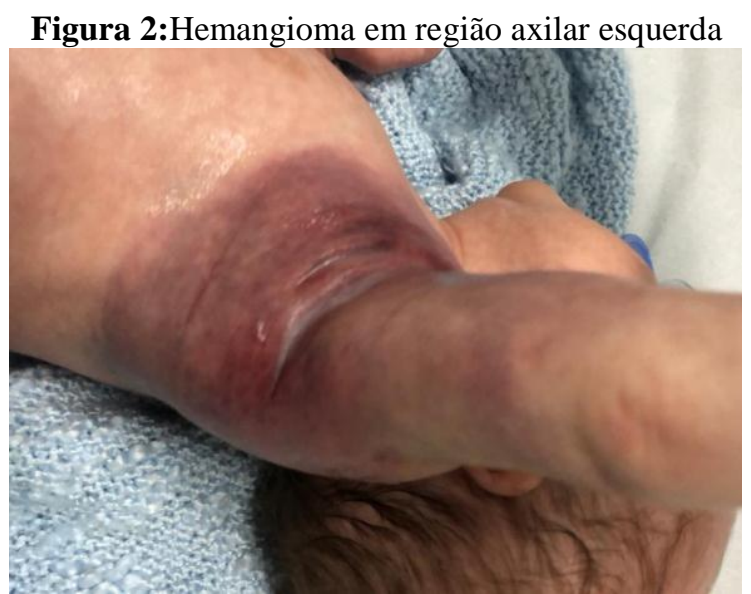

Fonte: Arquivo Pessoal

Durante todo o período, cuidadora negou demais queixas associadas. Foram realizados exames para triagem hematológica, que sugeria consumo de plaquetas. Nos gráficos demonstrados a seguir, foi evidenciado os valores dos exames solicitados durante o processo de seguimento do paciente, sendo possível notar melhora progressiva na contagem de plaquetas (gráfico 1), com valores atingindo a normalidade no início de fevereiro, bem como a presença de valores dentro da normalidade para níveis de protrombina $(70$ - 100\%), INR (gráfico 2) e dímeroD (gráfico 3).

Gráfico 1: Contagem de Plaquetas x Data

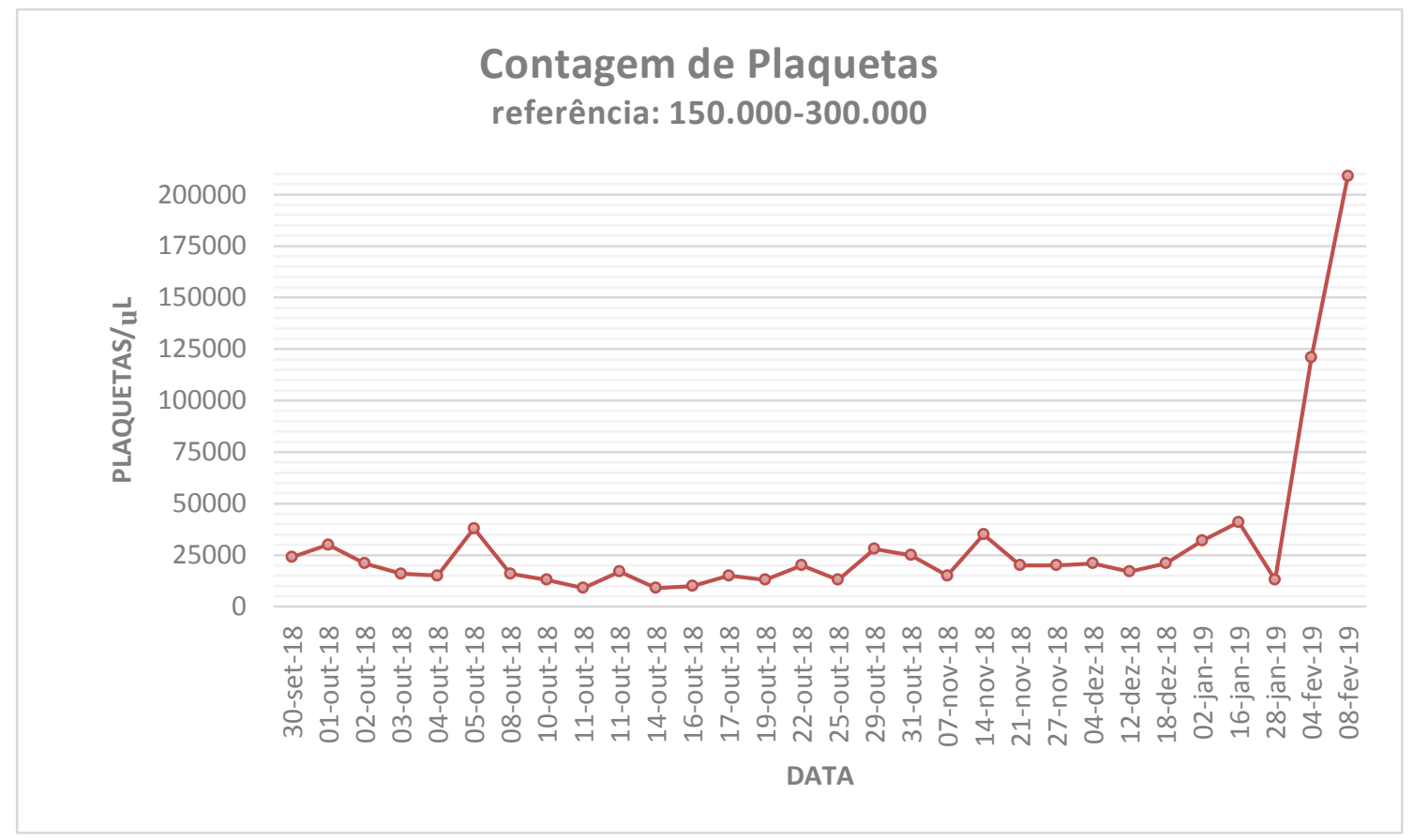

Fonte: elaborado pelo autor 
Gráfico2: Contagem Dímero D x Data

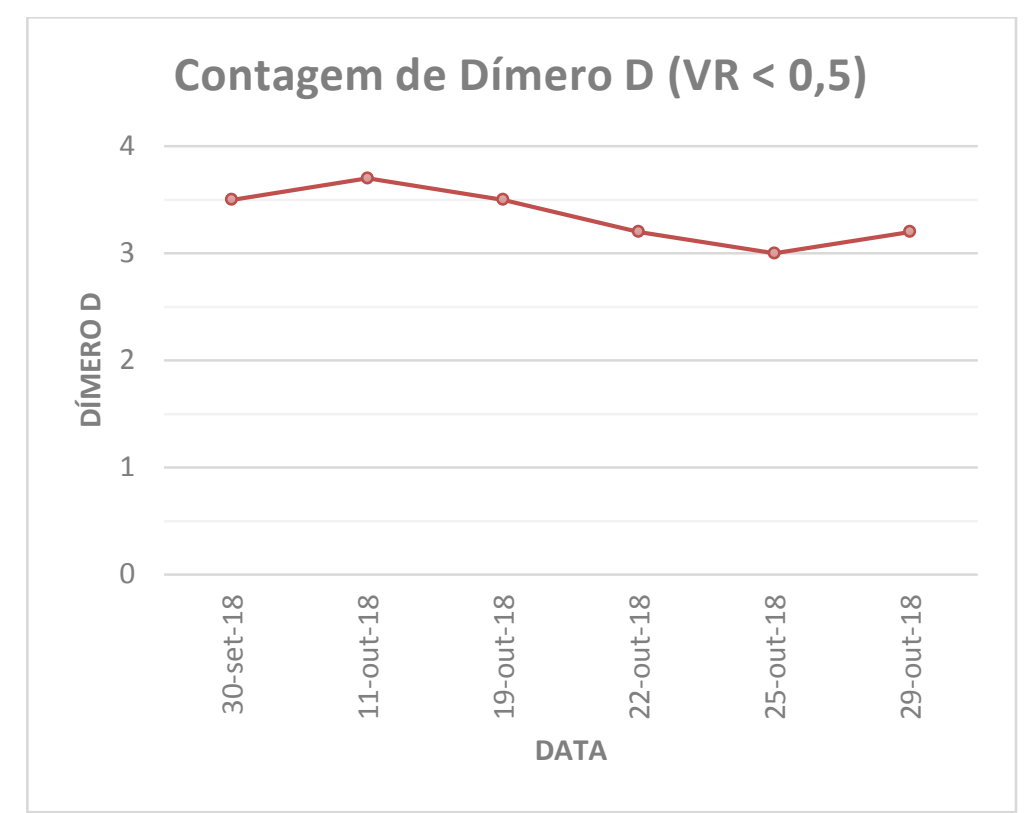

Fonte: elaborado pelo autor

Gráfico 3 - Atividade (\%) x Data e INR x Data

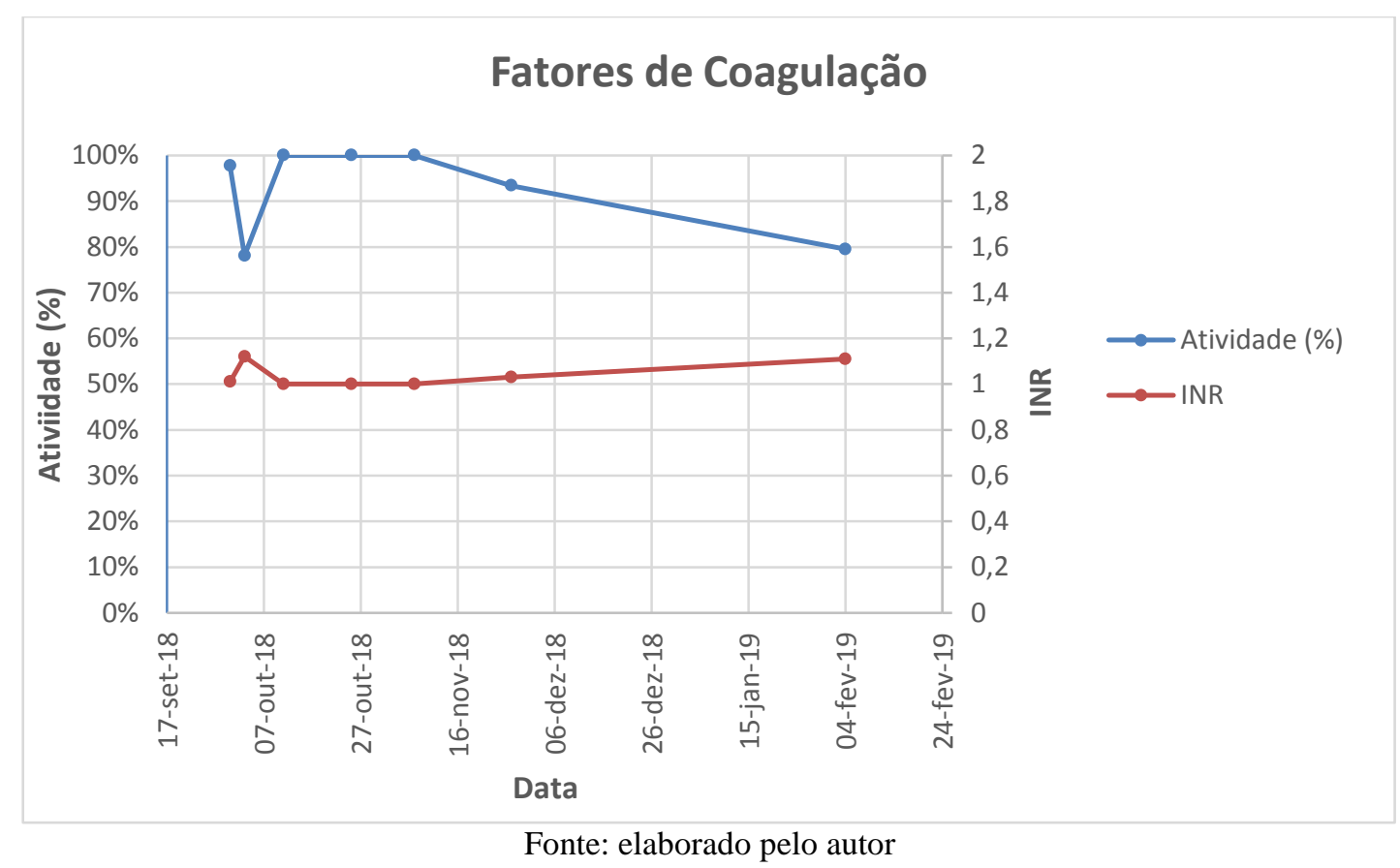

Paciente prematuro de 36 semanas e 3 dias. Mãe usuária de substâncias ilícitas e não realização de pré-natal. Em maternidade, VDRL materno positivo (1 / 64), sendo instituído tratamento para sífilis congênita provável (penicilina cristalina por 10 dias). Testes de triagem neonatal sem alterações.

Discutido caso com profissional médico vascular que juntamente com equipe levantou hipótese de fenômeno de Kasabach-Merrit. Iniciado neste momento tratamento com Prednisolona $3 \mathrm{mg} / \mathrm{kg} / \mathrm{dia}$. 
Foi necessário realizar transfusão de concentrado de hemácias e logo após, solicitada ultrassonografia associado ao doppler do membro acometido, em que ficou evidenciado edema de partes moles, porém, sem alterações de sistema venoso profundo, sem sinais de TVP.

No dia 12 de Outubro de 2018 optou-se por iniciar esquema de tratamento com propranolol, inicialmente a $1 \mathrm{mg} / \mathrm{kg} /$ dia e progredido até $3 \mathrm{mg} / \mathrm{kg} / \mathrm{dia}$. Manteve plaquetas variando de 10-15.000 (gráfico 1) com melhora no tempo de tromboplastina parcial ativada (TTPA) bem como dos valores de fibrinogênio (gráfico 5), sem aumento da lesão e sem sangramento ativo. Optado por consulta ambulatorial para seguimento do caso, recebendo alta hospitalar 14 dias após o início do tratamento com propranolol.

\section{Gráfico 4- TTPa x Data}

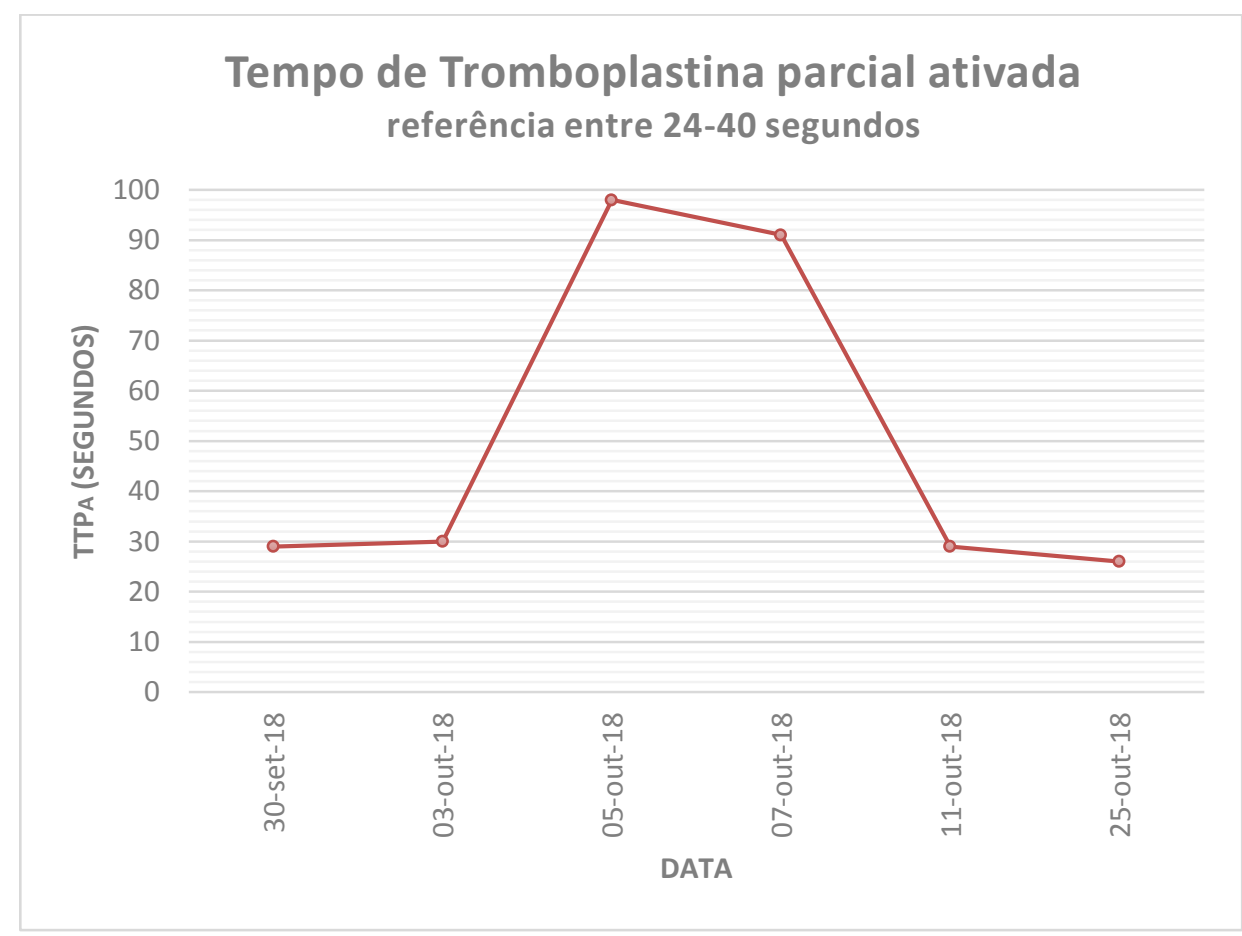

Fonte: elaborado pelo autor 
Gráfico 5- Contagem de Fibrinogênio x Data

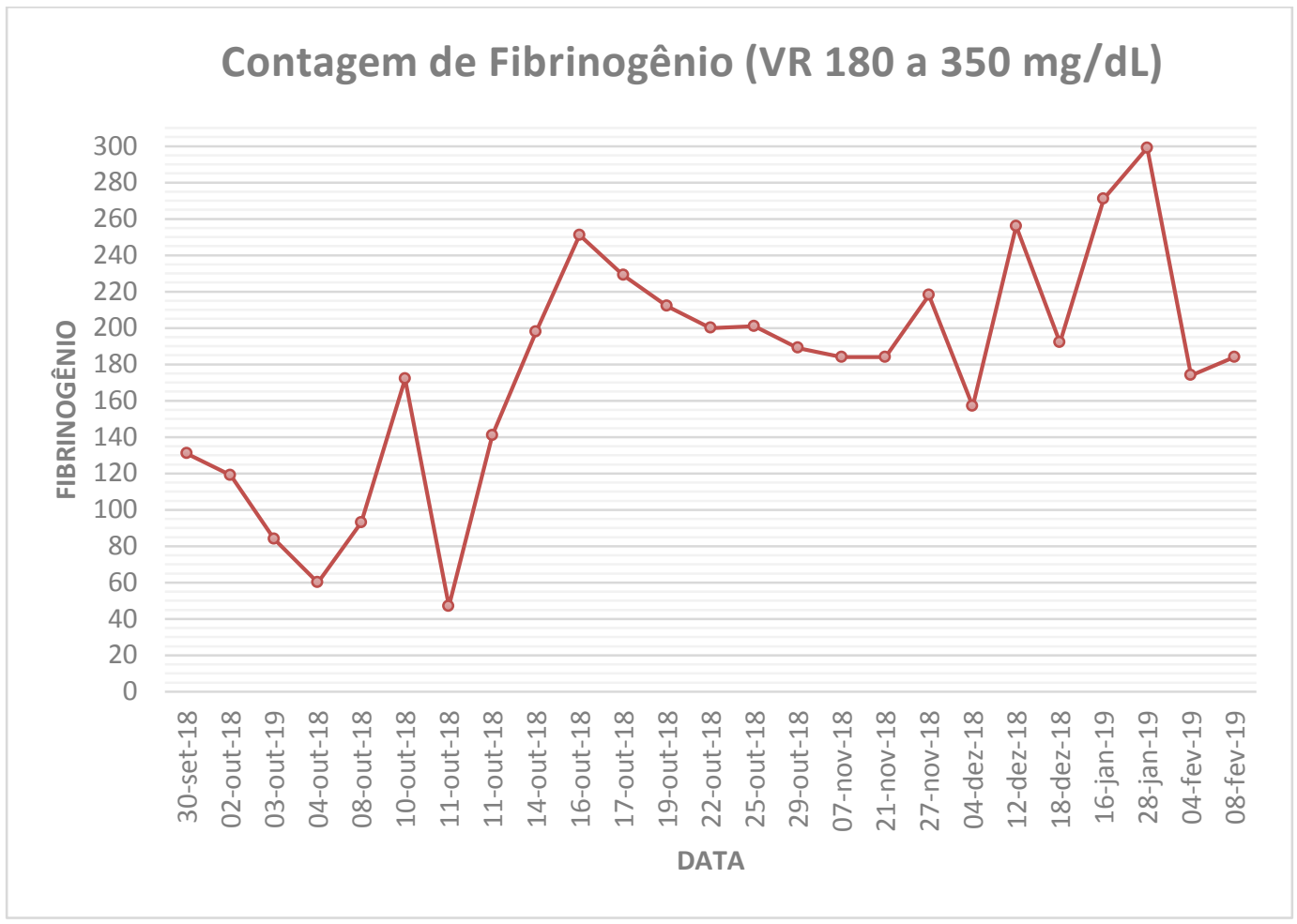

Fonte: elaborado pelo autor

Durante acompanhamento Ambulatorial, optado por desmame de Prednisolona conforme ocorria o aumento das doses de Propranolol, como evidenciado na tabela abaixo. Mesmo em dose terapêutica alta de Propranolol, paciente ainda apresentava manutenção de plaquetopenia (gráfico1). Optou-se por suspender gradativamente o Propranolol e iniciar Vincristina 0,5 $\mathrm{mg} / \mathrm{kg} / \mathrm{dose}$, uma vez por semana, obtendo-se boa resposta - melhora das plaquetas, diminuição gradativa do edema e hematoma. Segue em acompanhamento ambulatorial mensal, com boa resposta clínica até o momento.

Tabela 1- Duração do tratamento de acordo com o medicamento e sua respectiva posologia.

\begin{tabular}{|c|c|c|c|}
\hline MEDICAÇÃ O & POSOLOGIA & INÍCIO & TÉRMINO \\
\hline Prednisolona & $3 \mathrm{mg} / \mathrm{kg} / \mathrm{dia}$ & $01 / 10 / 18$ & $02 / 01 / 19$ \\
\hline Propranolol & $1 \mathrm{mg} / \mathrm{kg} / \mathrm{dia}$ & $12 / 10 / 18$ & $22 / 11 / 18$ \\
\hline Propranolol & $2 \mathrm{mg} / \mathrm{kg} / \mathrm{dia}$ & $22 / 11 / 18$ & $18 / 12 / 18$ \\
\hline Propranolol & $3 \mathrm{mg} / \mathrm{kg} / \mathrm{dia}$ & $18 / 12 / 18$ & $20 / 01 / 19$ \\
\hline Vincristina & $0,05 \mathrm{mg} / \mathrm{kg} / \mathrm{semana}$ & $24 / 01 / 19$ & $06 / 06 / 19$ \\
\hline
\end{tabular}

Fonte: elaborado pelo autor

\section{DISCUSSÃO}

Os hemangiomas, de forma geral apresentam história natural dividida em três fases. A primeira denominada de fase proliferativa é responsável pelo crescimento rápido do tumor podendo assumir grandes dimensões em relação ao tamanho da criança e comprometer o local em que se encontra, seja de forma funcional, estética ou até mesmo psíquica. A segunda fase é 
chamada de fase involutiva ou regressão espontânea sendo representada por $50 \%$ dos hemangiomas e em torno de $70 \%$ destes chegam até a terceira fase denominada involuída ou equilíbrio final. (HIRAKI; GOLDENBERG, 2018)

Os locais mais frequentes de aparecimento do hemangioma cutâneo são na cabeça, membros e tronco, porém também é possível encontrar essas lesões em órgãos internos, como fígado, baço e pulmão. Dessa forma, os sinais e sintomas que o paciente pode apresentar é a anemia, hepatomegalia e icterícia que, somados ao crescimento rápido do hemangioma, podem levar a formação de petéquias e equimoses. Tais manifestações são o que geralmente levantam suspeita diagnóstica, o que leva a realização de exames mais detalhados e a instauração de terapêutica (GONTIJO; SILVA; PEREIRA, 2003) (MUKAI; ZANLOCH et al., 2008).

Em vista de quadro, a Sindrome de Kasabach-Merrit é uma patologia que cursa com grande ameaça a vida, evoluindo com ativação plaquetária e seu posterior sequestro, aliado a diminuição dos fatores de coagulação pelo consumo da formação tumoral. Dessa forma, pode evoluir para complicações mais severas, como sangramentos espontâneos e coagulação intravascular disseminada (CIVD), sendo esse fator principal para evolução fatal da doença (GONTIJO; SILVA; PEREIRA, 2003).

Durante a primeira consulta do paciente do caso em questão, em ambiente ambulatorial, apresentava inicialmente lesão eritemato-violácea em região de ombro esquerdo $(+/ 4+)$, o que inicialmente foi questionado se não seria uma lesão ocorrida a partir de um trauma durante o parto. No entanto, não foi detectado alterações em movimentação do membro e ausência de fáceis de dor a mobilização. Contudo, tal quadro foi evoluindo sem melhora, com aparecimento repentino de edema violáceo no local, que se espalhou por quase todo o MSE.

Para o diagnóstico da síndrome de Kasabach-Merrit é necessário associar a história clínica do paciente com uso de exames laboratoriais, demonstrando presença de trombocitopenia profunda, normalmente abaixo de 20.000/mm3 e, a coagulopatia de consumo, expressa por hipofibrinogenemia e produtos de degradação da fibrina (dímero-D). Histologia, e exames de imagem, tais como ultrassom, ressonância magnética e angiografia digital podem auxiliar na confirmação do diagnóstico (BONINI; BELLODI; SOUZA, 2011); (BRONDANI et al., 2018). O exame de imagem, se torna de grande valia pois pode ser capaz de evidenciar possíveis complicações do quadro, como formação de trombose venosa profunda (TVP), o que seria responsável por agravar ainda mais a situação.

O paciente do caso foi abordado, inicialmente com plaquetopenia 24.000 chegando a níveis plasmáticos de 9.000 plaquetas/microlitros (Gráfico1), dímero D de 3,5 (Gráfico 2), INR 1,01 (Gráfico 3), TTPa de 29 segundos (Gráfico 4), e fibrinogênio $131 \mathrm{mg} / \mathrm{dl}$ (Gráfico 5), resultados esse compatíveis com o diagnóstico de SKM. No entanto, por ser uma síndrome rara, 
os diagnósticos diferenciais são imprescindíveis para auxiliar na escolha do plano terapêutico adequado, sendo assim é importante a diferenciação entre hemangioma e as manchas vasculares ou manchas salmão, malformações vasculares e outros tumores vasculares desencadeados na infância, tendo como base primordial a história clínica associada ao exame físico, condições essas mais comuns em pediatria (GONTIJO; SILVA; PEREIRA, 2003).

No cenário atual, não há consenso a respeito da terapia adequada para o tratamento de SKM. Dessa forma, é indicado o tratamento de suporte com transfusão de plaquetas, crioprecipitado e plasma fresco congelado (MUKAI et al., 2008). A literatura recomenda o uso de alguns medicamentos já reconhecidos para a síndrome, dentre eles, o corticóide sistêmico, como prednisolona $(2-3 \mathrm{mg} / \mathrm{kg} / \mathrm{dia})$, sendo este o mais comum utilizado, e pode ser administrado em posologias mais elevadas quando o paciente não se apresentar responsivo. Apesar da sua indicação rotineira de uso, possui efeitos adversos insidiosos difíceis de controlar, além de risco de infecções principalmente respiratórias. (MOURA et al., 2014)

Existem outras opções estudadas para o tratamento desse tipo de hemangioma como terapia compressiva, vincristina, ciclofosfamida, alfa-interferon, fármacos antifibrinolíticos e antiplaquetários, radioterapia localizada e embolizações. Vem crescendo, na atualidade, o uso de propranolol como uma das drogas alternativas, mostrando-se eficiente para alguns casos de hemangioma infantil, porém é necessário manter todos os pacientes em monitorização antes, durante e após tratamento, pois a medicação pode causar alguns efeitos já conhecidos, como bradicardia, hipertensão e hipoglicemia. (BONINI; BELLODI; SOUZA, 2011)

A dose recomendada para o propranolol é de $2 \mathrm{mg} / \mathrm{kg} / \mathrm{dia}$, porém é introduzida de forma gradual e em crianças menores que três meses de vida devem-se utilizar doses mais baixas, pois há risco de desenvolver hipoglicemia. Acredita-se que o mecanismo de ação do propranolol haja na redução do fator de crescimento básico de fibroblastos (bFGF) e também no fator de crescimento vascular do endotélio (VEGF) o que resulta em apoptose de células endoteliais, promovendo assim o não crescimento do tumor e sua diminuição de volume regularmente. (BONINI; BELLODI; SOUZA, 2011)

Embora as terapias medicamentosas ressaltadas apresentem grande eficiência no quadro de hemangiomas, a apresentação agressiva ou complicada, que normalmente não responde ao uso dessa terapêutica, é indicado iniciar um tratamento quimioterápico, sendo a vincristina usada como escolha e o interferon-alfa em desuso atualmente pelo risco de diplegia espástica, também conhecida como síndrome de Little em pacientes menores de um ano. Assim sendo, a vincristina está na categoria dos inibidores de microtúbulos, e possui uma atuação citotóxica associada à efeito contra-angiogênico através do bloqueio da proliferação endotelial e quimiotaxia, bem como suas interações com a fibronectina (GONTIJO; SILVA; PEREIRA, 2003). 
Dessa forma, é importante que cada caso seja individualizado, avaliado conforme sua gravidade, tentativas terapêuticas já utilizadas e evolução do quadro clínico. Pode ser necessário, em alguns pacientes, a instauração de procedimentos cirúrgicos, a fim de garantir a sobrevida, sendo estes realizados de duas principais formas: ressecção tumoral ou até mesmo amputação do membro acometido (MOURA et al., 2014)

\section{CONSIDERAÇÕES FINAIS}

A síndrome de Kasabach - Merrit é uma patologia rara que ocorre de forma mais prevalente na população pediátrica. Seu diagnóstico e tratamento, mesmo que ainda não bem definidos, quando instituídos de maneira precoce, previnem a progressão para complicações, que incluem plaquetopenia severa, trombose local e até mesmo o óbito, sendo imprescindível o reconhecimento, pelo médico, desta patologia.

O caso descrito demonstra a evolução de um paciente em que se realizou terapia inicial para hemangioma, o que auxiliou para não evolução para quadros mais graves. E ainda demostra que, mesmo frente ao diagnóstico e tratamento oportunos progrediu em intensidade apesar de terapia padrão básica (propranolol e corticoterapia).

\section{REFERÊNCIAS}

BONINI, F. K.; BELLODI, F. S.; SOUZA, E. M. Hemangioma infantil tratado compropranolol. Anais Brasileiros de Dermatologia, v. 86, n. 4, p. 763-766, 2011.

BRONDANI, T. G. et al. Hemangioma gigante e Síndrome De Kasabach Merrit. Boletim Científico de Pediatria, v. 7, n. 1, p. 47, 2018.

GONTIJO, B.; SILVA, C. M. R.; PEREIRA, L. B. Hemangioma da infância. Anais Brasileiros de Dermatologia, v. 78, n. 6, p. 651-673, 2003.

HAISLEY-ROYSTER, C. et al. Kasabach-Merritt phenomenon: a retrospective study of treatment with vincristine. Journal of Pediatric Hematology/Oncology, v. 24, n. 6, p. 459-62, setembro/agosto 2002.

HIRAKI, P. Y.; GOLDENBERG, D. C. Diagnóstico e tratamento do hemangioma infantil. Revista Brasileira de Cirurgia Plástica, v. 25, n. 2, 2018.

MOURA, R. et al. Síndrome de Kasabach-Merritt: tratamento clínico versus cirúrgico. Jornal Vascular Brasileiro, Botucatu, v. 13, n. 4, p. 330-335, outubro/dezembro 2014. 
MUKAI, A. D. O. et al. Hemangioendotelioma kaposiforme e síndrome de Kasabach-Merritt. Revista paulista de pediatria, São Paulo, v. 26, n. 2, p. 192-196, Junho 2008.

MUÑOZ, N. A. et al. Síndrome de Kasabach- Merrit. Revista Pediatría Electrónica, v. 15, n. 3, p. 7-12, 2018.

RYAN, C. et al. Kasabach-Merritt phenomenon: a single centre experience. European Journal of Haematology, v. 84, p. 97-104, outubro 2009.

SOCIEDADE BRASILEIRA DE PEDIATRIA. Hemangioma: Nova Classificação e Atualização Terapêutica.. 2018 Novembro; No 3. Departamento Cientifico de Dermatologia - SBP, n. 3, Novembro 2018.

YADAV, D. et al. 4. Yadav D, Maheshwari A, Aneja S, Seth A, Chandra J. Neonatal KasabachMerritt phenomenon. Indian Journal of Medical and Paediatric Oncology, v. 32, n. 4, p. 238241, outubro/novembro 2011. 